\title{
Gestational carriers: A viable alternative for women with medical contraindications to pregnancy ${ }^{*}$
}

\author{
Raymond M. Anchan ${ }^{1 \# \dagger}$, Stacey A. Missmer ${ }^{1,2,3 \#}$, Katharine F. Correia ${ }^{1}$, Elizabeth S. Ginsburg ${ }^{1}$ \\ ${ }^{1}$ Department of Obstetrics, Gynecology and Reproductive Biology, Brigham \& Women’s Hospital and Harvard Medical School, \\ Boston, USA \\ ${ }^{2}$ Channing Laboratory, Department of Medicine, Brigham \& Women’s Hospital and Harvard Medical School, Boston, USA \\ ${ }^{3}$ Department of Epidemiology, Harvard School of Public Health, Boston, USA \\ Email: ${ }^{\dagger}$ ranchan@partners.org
}

Received 9 May 2013; revised 10 June 2013; accepted 17 June 2013

Copyright (C 2013 Raymond M. Anchan et al. This is an open access article distributed under the Creative Commons Attribution License, which permits unrestricted use, distribution, and reproduction in any medium, provided the original work is properly cited.

\begin{abstract}
Objective: Compare the efficacy of surrogate or gestational carrier (GC) cycles to that of autologous in vitro fertilization (IVF)/intracytoplasmic sperm injections (ICSI) in patients with gynecologic or medical co-morbidities contraindicative to pregnancy. Design: Retrospective cohort study. Setting: Infertility patients from a single university hospital-based program from 1998-2009. Intervention(s): 128 GC cycles from 80 intended parents were identified and compared with 15,311 IVF or ICSI cycles. Main Outcome Measure(s): The peak estradiol (E2), number of oocytes retrieved, cycle cancellation, ongoing pregnancy, and live-birth were compared between GCs and autologous IVF carriers. Indications for GC use were also identified. Multiple cycles contributed by the same patient were accounted for using multivariable generalized estimating equations and two-sided Wald p-values. Results: Uterine factors (67\%) was the most common indication for using a GC, followed by non-gynecologic medical conditions including coagulopathies $(13 \%)$, end stage renal disease $(10 \%)$, cardiovascular disease (5\%) and cancer (5\%). Adjusting for age, ovulation induction in GC cycles had similar peak E2 levels and number of oocytes retrieved relative to IVF cycles ( $p=0.23$ and 0.43 , respectively). Clinical pregnancy $(49 \%$ vs. $42 \%, p=0.28)$ and live-birth rates $(31 \%$ vs. $32 \%, p=0.74)$ were also comparable. A sub-analysis of GC cycles in those women with uterine factor indications, demonstrated significantly higher clinical pregnancy rates $(O R=$ 2.0 ; $C I=1.2-3.5$ ) with $60 \%$ greater odds of live-birth relative to IVF/ICSI cycles, however this odds was not
\end{abstract}

\footnotetext{
There is no external funding support to disclose for this investigation.

"Both lead authors contributed equally to this manuscript.

${ }^{\dagger}$ Corresponding author.
}

statistically significant for differences in live-birth (CI = 0.9 - 2.9). Conclusions: GCs are a viable alternative to start families for patients with medical co-morbidities precluding pregnancy.

Keywords: IVF; Donor Egg; Gestational Carriers; Gestational Surrogacy; Infertility; Medical Comorbidities

\section{INTRODUCTION}

The physiologic changes and demands associated with pregnancy place significant stress on women. In addition to increased demands on cardiovascular output, renal function and glucose metabolism, physical stresses associated with labor and delivery are better tolerated if the patient is healthy at conception. Childbearing is often deferred to later stages of life for a variety of reasons including primary or secondary infertility, iatrogenic infertility, non-obstetrical medical morbidity and socioeconomic conditions [1-12]. Accordingly there is a need for alternatives to conventional childbearing for establishing one's family to address many of the age related physiologic changes women undergo.

The most comprehensive data regarding GC pregnancies stem from the European experience [3,7,13,14]. Earliest reports indicated clinical pregnancy rates of $18 \%$ [13,15], in cycles utilizing gestational carriers. However the notion of employing the assistance of others to conceive is ancient and is seen in the practice of having concubines help infertile women bear children in several early cultures including the Romans, Greeks and Chinese [16]. By definition a surrogate mother is one who provides her oocytes as well as uterus for the pregnancy [1, 13], while a gestational carrier is an individual who carries a non-biologic child for another intended parent (IP) 
and therefore only has a gestational relationship to the child $[1,9,15]$. The pregnancy thus is a result of an in vitro fertilization process using the IP's gametes and then transferring the embryo(s) to the GC's uterus. If the IP is unable to safely carry a pregnancy due to co-morbid conditions such as cardiac disease, severe osteoporosis, endometrial scarring from radiation therapy or surgery related to cancer, as examples, the use of a GC affords her the opportunity to have a genetically related child.

While the notion of using gestational carriers (GCs) is an attractive and seemingly safe option, there is a paucity of data examining the delivery success rates, because of the pregnancy or impact on the GC. In this study we examine the increasing practice of GC pregnancies and evaluate the efficacy of GCs in assisted reproductive technology.

\section{MATERIALS AND METHODS}

We conducted a retrospective cohort study including IVF cycles from 1998-2009 at a single university hospitalbased infertility clinic with Institutional Review Board (IRB) approval (IRB \# 2011p001000). During this 12 year period, 128 GC cycles were identified from 80 intended parents. The comparison group included 15,311 fresh IVF cycles from 7780 different women during this same time period. Cycles employing GIFT, ZIFT, oocyte donation, day 5 embryo transfer or cryo-embryo transfers were excluded. Cycles for women $<25$ years old and those who used a gestational carrier electively for non-medical indications (social reasons i.e. same sex couples) were also excluded from the study cohort Criteria for IVF acceptance are: day 3 FSH and E2, TSH $<3.0$ $\mathrm{mIU} / \mathrm{L}$, normal uterine cavity for non-GC cycles. (There is no absolute FSH cut off in our program, however women with FSH levels > $10 \mathrm{IU} / \mathrm{L}$ generally underwent poor responder protocols). Stimulation protocols used included a long leuprolide down regulation protocol, GnRH antagonist protocol which included 7 - 21 days of oral contraceptive pretreatment, and for poor responder's microflare or estrogen priming-antagonist stimulations. Stimulation included with 150 - 600 IU FSH per day starting dose, with or without additional LH which was administered as a human menopausal gonadotropin product. With the exception of three GCs who were 40.5, 40.7 , and 42.2 years old, all of the remaining gestational carriers in this analysis were less than 40 years old and must have had at least one term birth, no medical problems other than treated hypothyroidism, no history of miscarriage, or uterine surgery other than prior Cesarean section. Required FDA testing was rigorously adhered to in all cycles starting in 2005. These tests include immune screening for hepatitides (hepatitis B Surface antigen and hepatitis C core antibody), HTLV 1\&2, HIV 1\&2, Chla- smydia, Gonorrhea, Syphilis (RPR), CMV (IgG, IgM), rubella and PAP smear screening.

\section{OUTCOME DEFINITIONS}

An ongoing pregnancy included patients who were discharged from the IVF practice to their Obstetrician or Gynecologist's care with an intrauterine pregnancy detected via ultrasound with a fetal heart rate between 7 - 8 weeks of gestation. Pregnancy losses $<12$ weeks of gestation, chemical pregnancies and blighted ova were not considered to be ongoing pregnancies. Live-birth was defined as the birth of a live born infant at $>20$ weeks. Ongoing pregnancy and live-birth rates are reported per embryo transfer unless otherwise stated. A cancelled cycle indicated a poor response to hormonal stimulation during the IVF cycle and no oocyte retrieval or embryo transfer. Freeze all cycles were considered to be cancelled cycles and as previously indicated, cryo-embryo transfer cycles were excluded from the analysis.

\section{STATISTICAL ANALYSIS}

Generalized estimating equations that calculated odds ratios (OR) and 95\% confidence intervals (CI) were used to account for the correlation between cycles with embryos from the same intended parents. The normal distribution with identity link was specified when studying peak estradiol, a Poisson distribution with log link for number of oocytes retrieved, and a binomial distribution with logit link for cycle cancellation, ongoing pregnancy, and live-birth. Continuous age in months was adjusted for in all models a priori. Peak estradiol (E2) was additionally adjusted for when evaluating number of oocytes retrieved, as the effect estimate changed by greater than $10 \%$ when peak E2 was added to the model. The addition of peak E2 and number of embryos transferred (ET) changed the effect estimate for post-ET outcomes negligibly, and therefore are not included in the final models for pregnancy and live-birth. All Wald p-values are 2-sided with $<0.05$ considered to be statistically significant. Analyses were performed using Statistical Analysis Software $\left(\mathrm{SAS}^{\circledR}\right.$ ) version 9.1 (SAS Institute, Inc., Cary, NC).

\section{RESULTS}

A total of 128 GC cycles from 80 intended parents and 15,311 comparison IVF cycles from 7780 women were included in the study population. The age distribution was similar between groups, with a median age of 36.6 years among GC cycle intended parents (interquartile range, Q1 - Q3: 32.7 - 39.0) and 36.7 years among IVF cycles (Q1 - Q3: 33.6 - 39.6) (Table 1). Gestational carriers were between 21.6 and 42.2 years old, with a median age of 32.1 years (Q1 - Q3: 28.7 - 35.5). 
Table 1. Demographic characteristics by cycle type from a cohort of couples undergoing ART from 1998-2009 at a single university hospital-based infertility clinic.

\begin{tabular}{|c|c|c|}
\hline Characteristic & $\begin{array}{c}\text { IVF } \\
(n=15,311)\end{array}$ & $\begin{array}{l}\text { Gestational Carrier } \\
\qquad(\mathrm{n}=128)\end{array}$ \\
\hline \multicolumn{3}{|l|}{ Patient age } \\
\hline $\mathrm{N}$ & 15,311 & 128 \\
\hline Mean \pm SD & $36.4 \pm 4.0$ & $36.0 \pm 4.1$ \\
\hline Minimum-Maximum & $25.0-43.9$ & $27.2-43.9$ \\
\hline \multicolumn{3}{|l|}{ Gestational carrier age } \\
\hline $\mathrm{N}$ & - & 122 \\
\hline Mean \pm SD & - & $32.0 \pm 4.6$ \\
\hline Minimum-Maximum & & $21.6-42.2$ \\
\hline \multicolumn{3}{|l|}{ Cycle attempt number } \\
\hline $\mathrm{N}$ & 15,311 & 128 \\
\hline Mean \pm SD & $1.9 \pm 1.2$ & $2.4 \pm 1.8$ \\
\hline Minimum-Maximum & $1-10$ & $1-9$ \\
\hline \multicolumn{3}{|l|}{ Number of stimulation days } \\
\hline $\mathrm{N}$ & 15,253 & 128 \\
\hline Mean \pm SD & $11.1 \pm 2.1$ & $11.8 \pm 2.4$ \\
\hline Minimum-Maximum & $1-28$ & $5-22$ \\
\hline \multicolumn{3}{|l|}{ Total FSH (IU) } \\
\hline $\mathrm{N}$ & 15,253 & 128 \\
\hline Mean \pm SD & $3330.0 \pm 1868.5$ & $3623.8 \pm 1970.1$ \\
\hline Minimum-Maximum & $187.5-11700.0$ & $337.5-11400.0$ \\
\hline \multicolumn{3}{|c|}{ Number of embryos transferred } \\
\hline $\mathrm{N}$ & 13,579 & 113 \\
\hline Mean \pm SD & $3.0 \pm 1.6$ & $3.1 \pm 1.4$ \\
\hline Minimum-Maximum & $0-11$ & $0-9$ \\
\hline
\end{tabular}

The most common indication for using a gestational carrier was uterine factors (67\%), followed by coagulopathies (13\%), end stage renal disease (10\%), cardiovascular disease (5\%) and history of cancer (5\%). The comparison IVF cycles consisted of few uterine-factor infertility diagnoses (3\%). Other infertility diagnoses among the comparison group included tubal factor (18\%), ovulatory dysfunction (6\%), PCOS (4\%), male factor (28\%), other female factor (20\%), and unexplained (22\%) (Table 2).

GC cycles were stimulated similarly to the comparison IVF cycles. Both groups were stimulated for 11 days on average, with the GC group receiving a median of 3600 IU of gonadatropins (Q1 - Q3: 2100 - 4800) and the IVF group receiving a median of $3000 \mathrm{IU}$ of gonadatropins (Q1 - Q3: 1800 - 4800 Compared to women undergoing IVF alone, the odds of cycle cancellation among women using GCs was not significantly different (OR = 1.45; $95 \%$ CI $=0.71$ - 2.94) (Table 3). Peak E2 and number of oocytes retrieved were also similar between groups (Wald, two-sided p-value $=0.23$ and 0.43 , respectively).

Pregnancy and live-birth rates also were comparable between the two groups. Among embryo transfers, GC cycles had 1.2 times the odds of ongoing pregnancy
$(95 \%$ CI $=0.84-1.84)$ and 0.93 times the odds of live-birth (95\% CI $=0.61-1.43)$, as compared to IVF cycles. Results were similar when looking at the overall ongoing pregnancy and live-birth rates per cycle start (data not shown).

It is possible that the effect of utilizing a GC on cycle outcomes is different depending on IP age, though this study was not powered to detect heterogeneity between age strata. Descriptive statistics seem to suggest that the considerable decrease in ongoing pregnancy rates with increased age that is well established among traditional IVF cycles may not have as great an impact in GC cycles (Table 4(a)). However, live-birth rates were still considerably lower among intended parents in their late thirties and older. Again, small numbers make it difficult to draw definitive conclusions from these age-stratified analyses. A sub analysis was conducted comparing women with uterine indications in the GC group to those with uterine factor diagnoses in the comparison group (non-GC; Table 4(b)). Of the total analyzed cycles, three cycles $(\mathrm{n}=$ 86, $3.5 \%$ OR $=0.53,95 \% \mathrm{C}=0.15-1.81$ ) and thirty cycles ( $n=403,7.4 \%$ ) were cancelled in the GC and non-GC groups (Table $\mathbf{4 ( b ) )}$ respectively secondary to poor response to ovarian stimulation. Of the remaining 
Table 2. Descriptive statistics of cycle parameters and outcomes for 128 gestational carrier cycles, stratified by indication for gestational carrier use.

\begin{tabular}{|c|c|c|}
\hline & \multicolumn{2}{|c|}{ Indication for GC } \\
\hline & $\begin{array}{l}\text { Uterine Factor } \\
\quad(\mathbf{n}=\mathbf{8 6})\end{array}$ & $\begin{array}{l}\text { Medical Illness } \\
\qquad(\mathrm{n}=42)\end{array}$ \\
\hline \multicolumn{3}{|l|}{ IP Age } \\
\hline $\mathrm{N}$ & 86 & 42 \\
\hline Mean \pm SD & $36.3 \pm 3.8$ & $35.4 \pm 4.6$ \\
\hline Minimum-Maximum & $27.2-43.9$ & $28.8-43.8$ \\
\hline \multicolumn{3}{|c|}{ Number of stimulation days } \\
\hline $\mathrm{N}$ & 86 & 42 \\
\hline Mean \pm SD & $11.7 \pm 2.3$ & $11.8 \pm 2.5$ \\
\hline Minimum-Maximum & $5-22$ & $7-19$ \\
\hline \multicolumn{3}{|l|}{ Total IU Of FSH } \\
\hline $\mathrm{N}$ & 86 & 42 \\
\hline Mean \pm SD & $3616 \pm 2117$ & $3639 \pm 1654$ \\
\hline Minimum-Maximum & $450.0-11400$ & $337.5-6600$ \\
\hline \multicolumn{3}{|l|}{ Peak estradiol } \\
\hline $\mathrm{N}$ & 86 & 40 \\
\hline Mean \pm SD & $2068 \pm 997.7$ & $2028 \pm 1113$ \\
\hline Minimum-Maximum & $226-4708$ & $33-4117$ \\
\hline \multicolumn{3}{|c|}{ Number of oocytes retrieved } \\
\hline $\mathrm{N}$ & 86 & 42 \\
\hline Mean \pm SD & $14.6 \pm 8.2$ & $11.6 \pm 8.1$ \\
\hline Minimum-Maximum & $0-37$ & 0 - 32 \\
\hline \multicolumn{3}{|c|}{ Number of embryos transferred } \\
\hline $\mathrm{N}$ & 79 & 32 \\
\hline Mean \pm SD & $3.3 \pm 1.4$ & $3.0(1.0)$ \\
\hline Minimum-Maximum & $2-9$ & $2-6$ \\
\hline \multicolumn{3}{|l|}{ Any embryos frozen } \\
\hline $\mathrm{N}$ & $24(27.9 \%)$ & $14(33.3 \%)$ \\
\hline \multicolumn{3}{|l|}{ Cancelled cycle } \\
\hline $\mathrm{N}$ & $3(3.5 \%)$ & $7(16.7 \%)$ \\
\hline \multicolumn{3}{|l|}{ Embryo transfer } \\
\hline $\mathrm{N}$ & 79 (91.9\%) & $32(76.2 \%)$ \\
\hline \multicolumn{3}{|c|}{ Clinical pregnancy per cycle } \\
\hline $\mathrm{N}$ & 37 (43.0\%) & 17 (40.5\%) \\
\hline \multicolumn{3}{|c|}{ Clinical pregnancy per ET } \\
\hline $\mathrm{N}$ & 37 (46.8\%) & 17 (53.1\%) \\
\hline \multicolumn{3}{|l|}{ Live birth per cycle } \\
\hline $\mathrm{N}$ & $22(27.2 \%)$ & $10(26.3 \%)$ \\
\hline \multicolumn{3}{|l|}{ Live birth per ET } \\
\hline $\mathrm{N}$ & 22 (29.7\%) & 10 (35.7\%) \\
\hline
\end{tabular}

completed cycles the odds of ongoing pregnancy were statistically significantly 2-fold greater among women who used GCs (OR $=2.03$, 95\% CI = 1.18 - 3.49), however the 1.6 times greater odds of live-birth among the GC cycles did not reach statistical significance (95\% CI $=0.91-2.89$ ). This may be due to inadequate power to identify a true difference due to the small sample size.

Age stratified clinical outcomes among the women with uterine factor diagnoses (Table 4(b)) was also examined. As in the main analysis, there was some evidence of differential effect of GC by age, suggesting greater benefit of employing a GC among younger women with little benefit among older women-although the numbers are quite small within age-specific strata. Descriptively, ongoing pregnancy rates between groups appear more discrepant among the younger age groups. 
Table 3. Cycle outcomes by gestational carrier use within a cohort of couples undergoing IVF at a university hospital-based infertility clinic.

\begin{tabular}{|c|c|c|}
\hline Outcome & $\operatorname{IVF}(n=15,311)$ & Gestational Carrier $(n=128)$ \\
\hline \multicolumn{3}{|l|}{ Cancelled cycle $^{\mathrm{a}}$} \\
\hline n (\%) & $876(5.7 \%)$ & $10(7.8 \%)$ \\
\hline OR (95\% CI) & 1.00 (Ref) & $1.45(0.71-2.94)$ \\
\hline \multicolumn{3}{|l|}{ Peak estradiol $^{\mathrm{b}}$} \\
\hline $\begin{array}{c}\mathrm{N} \\
\text { Mean } \pm \text { SD } \\
\text { Median (Q1 - Q3) }\end{array}$ & $\begin{array}{c}14,517 \\
1928.3 \pm 977.1 \\
1758.0(1194.0-2528.0)\end{array}$ & $\begin{array}{c}126 \\
2055.6 \pm 1031.5 \\
1881.5(1322.0-2780.0)\end{array}$ \\
\hline p-value & - & 0.23 \\
\hline \multicolumn{3}{|l|}{ Number of oocytes retrieved ${ }^{c}$} \\
\hline $\begin{array}{c}\mathrm{N} \\
\text { Mean } \pm \text { SD } \\
\text { Median (Q1 - Q3) }\end{array}$ & $\begin{array}{c}14,385 \\
13.6 \pm 8.1 \\
12.0(8.0-18.0)\end{array}$ & $\begin{array}{c}128 \\
13.6 \pm 8.3 \\
13.0(6.5-19.5)\end{array}$ \\
\hline $\mathrm{p}$-value, test for linear trend & - & 0.43 \\
\hline \multicolumn{3}{|l|}{ Clinical pregnancy per cycle ${ }^{a}$} \\
\hline n (\%) & $5703(37.2 \%)$ & $54(42.2 \%)$ \\
\hline OR (95\% CI) & 1.00 (Ref) & $1.19(0.82-1.73)$ \\
\hline \multicolumn{3}{|l|}{ Clinical pregnancy per $\mathbf{E T}^{\mathrm{a}}$} \\
\hline n (\%) & 5703 (42.4\%) & $54(48.6 \%)$ \\
\hline OR (95\% CI) & 1.00 (Ref) & $1.24(0.84-1.84)$ \\
\hline \multicolumn{3}{|l|}{ Live-birth per cycle $^{\text {a }}$} \\
\hline n (\%) & $4161(28.1 \%)$ & 32 (26.9\%) \\
\hline OR (95\% CI) & 1.00 (Ref) & $0.90(0.60-1.37)$ \\
\hline \multicolumn{3}{|l|}{ Live-birth per $\mathbf{E T}^{\mathrm{a}}$} \\
\hline n (\%) & 4161 (32.1\%) & 32 (31.4\%) \\
\hline OR (95\% CI) & 1.00 (Ref) & $0.93(0.61-1.43)$ \\
\hline
\end{tabular}

${ }^{\mathrm{a}}$ Odds ratios (OR) and 95\% confidence intervals (CI) are from generalized estimating equations accounting for correlation between cycles from the same woman, with a binomial outcome distribution and a logit link. Models are adjusted for continuous age. ET = embryo transfer, Clinical pregnancy = Ultrasound documented intrauterine pregnancy with fetal heart rate between 7 - 8 weeks gestation. ${ }^{b}$ p-values are from generalized estimating equations accounting for correlation between cycles from the same woman, with a normal outcome distribution and the identity link. Models are adjusted for continuous age. 'p-values are from generalized estimating equations accounting for correlation between cycles from the same woman, with a Poisson outcome distribution and a log link. Models are adjusted for continuous age and peak E2. For GC cycles, age is that of the intended parent.

Among patients aged 25 to 30, 23\% (6/27) of comparison cycles versus $75 \%$ (3/4) of gestational carrier cycles had live-births; among patients over 35 years old, 17\% (37/228) of comparison cycles and 18\% (8/47) of gestational carrier cycles had live-births.

\section{DISCUSSION}

The indications for using a gestational carrier (GC) for pregnancy can be highly varied ranging from general medical conditions to congenital mullerian anomalies that preclude pregnancy (Table 2) [1-6,11,12]. While such health issues may not allow a woman to carry her own pregnancy, the patients' overall gonadal fertility may be quite normal. With the wide spread availability of in vitro fertilization, the practice of employing GCs is becoming increasingly common. Furthermore the clinical indications for using a GC continue to expand. Accordingly it is important to better understand the efficacy of this treatment in different populations of intended parents. Additionally it is of interest to determine the relative contribution of the uterine environment to the success of a pregnancy independent of the IVF stimulation cycle.

Despite the small number of GC attempts in this study, to our knowledge this is currently one of the largest retrospective cohort studies that evaluate the efficacy of 
Table 4. Descriptive statistics of cycle outcomes stratified by gestational carrier use and age at cycle start ${ }^{\mathrm{a}}$.

\begin{tabular}{|c|c|c|c|c|c|c|c|}
\hline \multirow[t]{3}{*}{$4(a)$} & \multicolumn{7}{|c|}{ All cycles } \\
\hline & \multicolumn{2}{|c|}{25 - 30 years old } & \multicolumn{2}{|c|}{31 - 35 years old } & \multicolumn{3}{|c|}{$36+$ years old } \\
\hline & $\operatorname{IVF}(n=1594)$ & GC $(n=13)$ & $\operatorname{IVF}(n=5058)$ & \multirow{2}{*}{$\begin{array}{c}\text { GC }(\mathrm{n}=46) \\
3(6.5 \%)\end{array}$} & \multicolumn{2}{|c|}{$\operatorname{IVF}(\mathrm{n}=8659)$} & GC $(n=69)$ \\
\hline Cancelled cycle & $48(3.0 \%)$ & $1(7.7 \%)$ & 228 (4.5\%) & & 600( & & $6(8.7 \%)$ \\
\hline Peak estradiol & $2114 \pm 1025$ & $1866 \pm 1141$ & $1999 \pm 977$ & $2314 \pm 1000$ & 1851 & & $1914 \pm 1011$ \\
\hline Oocytes retrieved & $16.8 \pm 8.9$ & $14.2 \pm 8.5$ & $14.8 \pm 8.2$ & $16.0 \pm 7.9$ & 12.3 & & $11.9 \pm 8.1$ \\
\hline $\begin{array}{l}\text { Clinical pregnancy per } \\
\text { ET }\end{array}$ & 740 (53.0\%) & $5(45.5 \%)$ & 2181 (48.5\%) & $22(55.0 \%)$ & 2782 & $\%)$ & 27 (45.0\%) \\
\hline \multirow[t]{2}{*}{ Live-birth per ET } & 595 (44.5\%) & $4(40.0 \%)$ & $1728(40.0 \%)$ & 15 (42.9\%) & 1838 & $\%)$ & $13(22.8 \%)$ \\
\hline & $\operatorname{IVF}(n=31)$ & GC $(n=5)$ & $\operatorname{IVF}(n=112)$ & $\mathrm{GC}(\mathrm{n}=31)$ & IVF (n & 60) & GC $(n=50)$ \\
\hline Cancelled cycle & $0(0 \%)$ & $0(0 \%)$ & $10(8.9 \%)$ & $2(6.5 \%)$ & $20(7$ & & $1(2.0 \%)$ \\
\hline $\begin{array}{l}\text { Clinical pregnancy per } \\
\text { ET }\end{array}$ & 7 (25.9\%) & $3(75.0 \%)$ & 33 (35.5\%) & 15 (53.6\%) & $61(2$ & & 19 (40.4\%) \\
\hline Live-birth per ET & $6(23.1 \%)$ & $3(75.0 \%)$ & $21(24.7 \%)$ & $11(44.0 \%)$ & $37(1$ & & $8(17.8 \%)$ \\
\hline \multirow[t]{2}{*}{$4(b)$} & \multicolumn{7}{|c|}{ Cycles with a uterine factor infertility diagnosis (GC cycles $n=86$ non-GC uterine factor cycles $n=403$ ) } \\
\hline & \multicolumn{2}{|c|}{$\operatorname{IVF}(n=31)$} & \multicolumn{2}{|c|}{$\operatorname{IVF}(\mathrm{n}=112)$} & GC $(\mathrm{n}=31)$ & $\operatorname{IVF}(n=260)$ & GC $(n=50)$ \\
\hline Cancelled cycle & \multicolumn{2}{|c|}{$0(0 \%)$} & \multicolumn{2}{|c|}{$10(8.9 \%)$} & $2(6.5 \%)$ & $20(7.7 \%)$ & $1(2.0 \%)$ \\
\hline Clinical pregnancy per & \multicolumn{2}{|c|}{7 (25.9\%) } & \multicolumn{2}{|c|}{33 (35.5\%) } & $15(53.6 \%)$ & $61(26.8 \%)$ & $19(40.4 \%)$ \\
\hline Live-birth per ET & \multicolumn{2}{|c|}{$6(23.1 \%)$} & \multicolumn{2}{|c|}{$21(24.7 \%)$} & $11(44.0 \%)$ & 37 (16.6\%) & $8(17.8 \%)$ \\
\hline
\end{tabular}

${ }^{a}$ Values presented are $\mathrm{n}(\%)$ for categorical variables and mean \pm SD for continuous variables. For GC cycles, the age is that of the intended parent.

GC-cycles in patients undergoing assisted reproductive treatment at a single university hospital-based infertility clinic. In this retrospective cohort study, we observed that patients using a gestational carrier demonstrate a similar response to ovarian stimulation relative to other infertile couples undergoing a typical IVF/ICSI cycle as reflected by peak estradiol levels, amount of hormonal stimulation required to elicit a maximal response and number of oocytes retrieved. These observations held true despite differences in stimulation protocols employed in these patients. In our study we did not segregate patients based on individual variations in race, ethnicity or other non-medical characteristics. The total number of embryos transferred in both groups was comparable. As anticipated the pregnancy success rates remained associated with the intended parent's age since this reflects the genetic contribution of the oocyte. These observations are consistent with several prior smaller studies that show GC live-birth rates to be higher than age-matched controls [1-3,7-10,13,15,17-25]. However in our study population, this difference was only evident among intended parents under age 35 .

The implications of this result are various, including the observation that there might be an unrecognized uterine component contribution to the infertility of patients who are diagnostically classified to have dimin- ished ovarian reserve, ovulatory dysfunction or unexplained infertility. Such a putative uterine factor may also be related to age-dependent intrinsic properties of the maternal endometrium affecting implantation, placental growth, function and perfusion or the maternal immune response thereby impacting pregnancy outcomes in the infertile population [26-29]. By employing a GC, one or more of these factors are altered resulting in improved ongoing pregnancy rates. Thus it might be more informative to compare GC delivery rates and outcomes to the general obstetrical population than the infertile population of patients. If indeed the uterine milieu is improved in GC pregnancies who were selected because of their proven successful obstetrical histories, one would predict that the fetal outcomes should also reflect this in terms of fetal weight, and gestational age at time of delivery. A per cycle (not per patient) retrospective cohort analysis from the Society for Assisted Reproductive Technologies (SART) data base [25] supports such a hypothesis.

In this retrospective analysis of the SART database looking at obstetrical outcomes from 2004-2006, differences in the uterine environment appeared to influence gestational age (GA) and birth weight (BW) with GC cycles faring better for BW than even donor egg (DE) cycles. This observation held true even when the uterine defect such as adhesions or leiomyomata were addressed 
prior to the initiation of fertility treatment. By contrast the GA in GCs was noted to be slightly less than either fresh IVF or DE cycles, consistent with the notion that the uterine environment might provide putative immunological factors influencing the duration of pregnancies. This latter result is curious and warrants further investigation.

In summary, our results demonstrate delivery rates comparable to that of the general IVF population when GCs are used to achieve a genetically related live-birth. Of equal importance is the observation that GC pregnancies are indeed a viable option for patient's with comorbidities that would preclude them from carrying their own pregnancy. Thus while there are a host of ethical and social concerns one must consider prior to using a GC [30-44], gestational surrogacy does appear to benefit patients wanting to start a family who themselves have obstetrical contraindications precluding them from becoming pregnant. Furthermore, the use of GCs in patients with uterine indications resulting in miscarriages and pregnancy loss may prove to have higher ongoing pregnancy and live-birth rates. Further studies are needed to investigate the exact nature of the beneficial factors the GC uterus may be providing to contribute to these favorable ART outcomes as well as better understand the immunologic interplay between the placenta and the endometrial lining of the host uterus. Our ongoing investigations will address the influence of obstetrical complications such as preeclampsia or gestational diabetes on GCs $[45,46]$, as such information would contribute to our understanding of the pathophysiology of these diseases and would be important to appropriately counsel intended parents and potential GCs regarding the risks and benefits of GC pregnancies.

\section{ACKNOWLEDGEMENTS}

RMA, SAM and KFC collected and analyzed data, RMA, SAM and ESG wrote and edited this manuscript. RMA and SAM had full access to data. None of the authors have any conflict of interests to declare. RMA and SAM contributed equally to this study.

\section{REFERENCES}

[1] Anchan, R.M. and Ginsburg, E.S. (2012) Gestational carrier pregnancy. In: Basow, D.S., Ed., UpToDate, Waltham, 2012

[2] Steigrad, S., Hacker, N.F. and Kolb, B. (2005) In vitro fertilization surrogate pregnancy in a patient who underwent radical hysterectomy followed by ovarian transposition, lower abdominal wall radiotherapy, and chemotherapy. Fertility and Sterilit, 83, 1547. doi:10.1016/i.fertnstert.2005.01.093

[3] Meniru, G.I. and Craft, I. (1997) Assisted conception options for patients with good-prognosis cervical cancer.
Lancet, 349, 542. doi:10.1016/S0140-6736(97)80093-1

[4] Siddle, N., Sarrel, P. and Whitehead, M. (1987) The effect of hysterectomy on the age at ovarian failure: Identification of a subgroup of women with premature loss of ovarian function and literature review. Fertility and Sterilit, 47, 94.

[5] Esfandiari, N., Claessens, E.A., O’Brien, A., et al. (2004) Gestational carrier is an optimal method for pregnancy in patients with vaginal agenesis (Rokitansky syndrome). International Journal of Fertility and Women's Medicine, 49, 79.

[6] Beski, S., Gorgy, A., Venkat, G., et al. (2000) Gestational surrogacy: A feasible option for patients with Rokitansky syndrome. Human Reproduct, 15, 2326-2328. doi:10.1093/humrep/15.11.2326

[7] Brinsden, P.R. (2003) Gestational surrogacy. Human Reproduct, 9, 483-491. doi:10.1093/humupd/dmg033

[8] Stafford-Bell, M.A. and Copeland, C.M. (2001) Surrogacy in Australia: Implantation rates have implications for embryo quality and uterine receptivity. Reproduction, Fertility and Development, 13, 99-104. doi:10.1071/RD00044

[9] Marrs, R.P., Ringler, G.E., Stein, A.L., et al. (1993) The use of surrogate gestational carriers for assisted reproductive technologies. American Journal of Obstetrics \& Gynecology, 168, 1858-1863. doi:10.1016/0002-9378(93)90702-K

[10] Farley, D.M., Grainger, D.A., Tjaden, B.L., et al. (2007) Use of a gestational carrier for a patient with recurrent adverse pregnancy outcomes from early onset severe preeclampsia. Fertility and Sterilit, 87, 189.

[11] Pellicer, A., Simón, C. and Remohí, J. (1995) Effects of aging on the female reproductive system. Human Reproduct, 10, 77-83. doi:10.1093/humrep/10.suppl_2.77

[12] Cano, F., Simón, C., Remohí, J. and Pellicer, A. (1995) Effect of aging on the female reproductive system: Evidence for a role of uterine senescence in the decline in female fecundity. Fertility and Sterilit, 64, 584.

[13] Utian, W.H., Goldfarb, J.M., Kiwi, R., et al. (1989) Preliminary experience with in vitro fertilization-surrogate gestational pregnancy. Fertility and Sterilit, 52, 633.

[14] Meniru, G.I. and Craft, I.L. (1997) Experience with gestational surrogacy as a treatment for sterility resulting from hysterectomy. Human Reproduct, 12, 51-54. doi:10.1093/humrep/12.1.51

[15] Utian, W.H., Sheean, L., Goldfarb, J.M. and Kiwi, R. (1985) Successful pregnancy after in vitro fertilization and embryo transfer from an infertile woman to a surrogate. The New England Journal of Medicine, 313, 13511352. doi:10.1056/NEJM198511213132112

[16] Marino, F. (2010) Global issues: Adoption and surrogate pregnancy. Infobase Publishing, New York.

[17] www.cdc.gov

[18] Braverman, A.M. and Corson, S.L. (1992) Characteristics of participants in a gestational carrier program. Journal of Assisted Reproduction and Genetics, 9, 353-357. doi:10.1007/BF01203959 
[19] Pirhonen, J., Bergersen, T.K., Abdlenoor, M., et al. (2005) Effect of maternal age on uterine flow impedance. Journal of Clinical Ultrasound, 33, 14-17. doi:10.1002/jcu.20079

[20] Brinsden, P.R., Appleton, T.C., Murray, E., et al. (2000) Treatment by in vitro fertilisation with surrogacy: Experience of one British centre. BMJ, 320, 924. doi:10.1136/bmj.320.7239.924

[21] Batzofin, J., Nelson, J., Wilcox, J., et al. (1999) Gestational surrogacy: Is it time to include it as part of ART? ASRM Annual Meeting Programme Supplement.

[22] Goldfarb, J.M., Austin, C., Peskin, B., et al. (2000) Fifteen years experience with an in-vitro fertilization surrogate gestational pregnancy programme. Human Reproduct, 15, 1075-1078. doi:10.1093/humrep/15.5.1075

[23] Raziel, A., Schachter, M., Strassburger, D., et al. (2005) Eight years' experience with an IVF surrogate gestational pregnancy programme. Reproductive BioMedicine Online, 11, 254-258. doi:10.1016/S1472-6483(10)60966-2

[24] Corson, S.L., Kelly, M., Braverman, A.M. and English, M.E. (1998) Gestational carrier pregnancy. Fertility and Sterilit, 69, 670-674. doi:10.1016/S0015-0282(98)00020-X

[25] Gibbons, W.E., Cedars, M. and Ness, R.B. and Society for Assisted Reproductive Technologies Writing Group (2011) Toward understanding obstetrical outcome in advanced assisted reproduction: Varying sperm, oocyte, and uterine source and diagnosis. Fertility and Sterilit, 95, 1645-1649. doi:10.1016/j.fertnstert.2010.11.029

[26] Ubaldi, F., Rienzi, L., Baroni, E., et al. (2003) Implantation in patients over 40 and raising FSH levels-A review. Placenta, 24, S34-S38. doi:10.1016/S0143-4004(03)00179-6

[27] Abdalla, H.I., Wren, M.E., Thomas, A. and Korea, L. (1997) Age of the uterus does not affect pregnancy or implantation rates; A study of egg donation in women of different ages sharing oocytes from the same donor. $\mathrm{Hu}$ man Reproduct, 12, 827-829. doi:10.1093/humrep/12.4.827

[28] Navot, D., Drews, M.R., Bergh, P.A., et al. (1994) Agerelated decline in female fertility is not due to diminished capacity of the uterus to sustain embryo implantation. Fertility and Sterilit, 61, 97.

[29] Brinsden, P.R. (1999) Surrogacy. In: Brinsden, P.R., Ed., A textbook of in vitro fertilization and assisted reproduction, Parthenon, Carnforth and New York.

[30] Cherry, A.L. (2011) Nurturing in the service of white culture: Racial subordination, gestational surrogacy, and the ideology of motherhood. Texas Journal of Women and the Law, 10, 83.

[31] Pretorius, D. (1994) Surrogate motherhood: A worldwide view of the issues. The New England Journal of Medicine, 331, 685.

[32] Golombok, S., MacCallum, F., Murray, C., et al. (2006) Surrogacy families: Parental functioning, parent-child re- lationships and children's psychological development at age 2. Journal of Child Psychology and Psychiatry, 47, 213-222. doi:10.1111/j.1469-7610.2005.01453.x

[33] Van Zyl, L. (2002) Intentional parenthood: Responsibilities in surrogate motherhood. Health Care Analysis, 10, 165-175. doi:10.1023/A:1016550002211

[34] Fuscaldo, G. (2006) Genetic ties: Are they morally binding? Bioethics, 20, 64-76. doi:10.1111/j.1467-8519.2006.00478.x

[35] Bravennan, A.M. and Corson, S.L. (2002) A comparison of oocyte donors' and gestational carriers/surrogates' attitudes towards third party reproduction. Journal of Assisted Reproduction and Genetics, 19, 462-469. doi:10.1023/A:1020306402235

[36] Gunn, W. (1994) Bioethics and law: Standard of care: The law of American bioethics. JAMA, 271, 795. doi:10.1001/jama.1994.03510340085047

[37] Yamamoto, K. and Moore, S.A. (2011) A trust analysis of a gestational carrier's right to abortion. Fordham Law Review, 70, 93.

[38] Rosen, A. (2005) Third-party reproduction and adoption in cancer patients. JNCI Monographs, 2005, 91-93. doi:10.1093/jncimonographs/lgi021

[39] MacCallum, F., Lycett, E., Murray, C., et al. (2003) Surrogacy: The experience of commissioning couples. $\mathrm{Hu}$ man Reproduct, 18, 1334-1342. doi:10.1093/humrep/deg253

[40] Massachusetts' Supreme Judicial Court (2001) Culliton v. Beth Israel Deaconess Medical Center. Wests North East Rep., 756, 1133-1141.

[41] Committee on Ethics (2008) ACOG committee opinion number 397, February 2008: Surrogate motherhood. $\mathrm{Ob}$ stetrics \& Gynecology, 111, 465.

[42] Guichon, J. (2007) The body, emotions and intentions: Challenges of preconception arrangements for health care providers. CMAJ, 176, 479-481. doi:10.1503/cmaj.061595

[43] Reilly, D.R. (2007) Surrogate pregnancy: A guide for Canadian prenatal health care providers. CMAJ, 176, 483-485. doi:10.1503/cmaj.060696

[44] Adams, K.E. (2003) Gestational surrogacy for a human immunodeficiency virus seropositive sperm donor: What are the ethics? Journal of the American Medical Women's Association, 58, 138.

[45] Duffy, D.A., Nulsen, J.C., Maier, D.B., et al. (2005) Obstetrical complications in gestational carrier pregnancies. Fertility and Sterilit, 83, 749-754. doi:10.1016/j.fertnstert.2004.08.023

[46] Parkinson, J., Tran, C., Tan, T., et al. (1999) Perinatal outcome after in-vitro fertilization-surrogacy. Human Reproduct, 14, 671-676. doi:10.1093/humrep/14.3.671 